\title{
Role of ultrasound and Doppler findings as a predictor of thyroid hormonal levels in cases of Hashimoto thyroiditis
}

Rasha Mahmoud ${ }^{1 *} \mathbb{D}$, Khaled Mohammed Azeem², Ahmed Safaa Ahmed Sayed ${ }^{3}$ and Faten Mohamed Ali ${ }^{1}$

\begin{abstract}
Background: Chronic lymphocytic thyroiditis or Hashimoto's thyroiditis (HT) is a common form of organ specific autoimmune disease mediated by anti-thyroid autoantibodies. Ultrasound including Doppler is helpful diagnostic tool in the diagnosis of Hashimoto's thyroiditis. This study aimed to evaluate the ultrasound and Doppler characters of Hashimoto thyroiditis and to analyze the relationship between these ultrasound, Doppler features and thyroid hormones level.

Results: Thyroid gland sonographic parenchymal abnormalities (septations, undulation, micronodularity, gland hyper vascularity, hypoechogenicity, sizable nodules as well as enlarged para-tracheal lymph nodes) were significantly higher in Hashimoto cases compared to control group ( $P$ value is $<0.001$ ). Undulation of thyroid gland margin was the most sensitive sonographic sign seen in about $72 \%$ of studied cases. Thyroid stimulating hormone (TSH) level was significantly high in cases with grade 2 echogenicity compared to cases with grade 0 and grade 1 echogenicity and was significantly high in cases with para-tracheal abnormal enlarged lymph nodes $(p<0.05)$. TSH \& thyroxine (T4) levels were significantly high in cases with high gland vascularity in Doppler study $(p<0.05)$.
\end{abstract}

Conclusions: Ultrasound is a very good diagnostic tool in $\mathrm{HT}$ with established signs. It can suggest hypothyroidism (high TSH) in cases of grade 2 parenchymal echogenicity, high vascularity and para-tracheal lymph nodes enlargement. These finding could be helpful in patient management and can contribute in better understanding the disease progression.

Keywords: Hashimoto thyroiditis, Ultrasound, Thyroid hormones, Autoimmune thyroiditis, Hypothyroidism

\section{Background}

Chronic lymphocytic thyroiditis or Hashimoto's thyroiditis (HT) is a common form of organ specific autoimmune disease mediated by anti-thyroid autoantibodies. It affects up to $2 \%$ of all females in the population and occurs mainly between the ages of 30 and 50 years [1].

Hashimoto's thyroiditis is one of the common causes of hypothyroidism, which may be subclinical in up to $90 \%$ of cases. However, mild thyrotoxicosis can be observed.

\footnotetext{
*Correspondence: dr-rasha.mahmoud@hotmail.com; dr.rasha. mahmoudgoma@gmail.com

1 Radiology Department, Bani-Seuf University, Bani Seuf, Egypt Full list of author information is available at the end of the article
}

Mild hypothyroidism may be present in $20 \%$ of patients when first seen or can develop over several years [2].

Ultrasound including Doppler is helpful diagnostic tool in the diagnosis of Hashimoto's thyroiditis [3]. Combination of different sonographic features such as echogenicity, echotexture, vascularity, gland diameters, and glandular margin has high sensitivity and specificity in HT diagnosis [4].

\subsection{Aims}

To evaluate the ultrasound \& Doppler characters of Hashimoto thyroiditis and to analyze the relationship between these ultrasound, Doppler features and thyroid hormones level. 


\section{Patients and methods}

\subsection{Study approval}

This study used the data on PACS \& the study protocol subjected to approval by the ethical committee of the faculty of medicine.

\subsection{Study design}

A cross-sectional randomized case-control study was conducted.

\subsection{Patients}

Clinical and ultrasonographic data of recorded studies on PACS of 97 patients of both sexes who pathologically proved to have Hashimoto thyroiditis who were referred to radiology department from January 2015 to December 2020 for ultrasound examination of thyroid gland. According to patient levels of TSH, T4 \& triiodothyronine (T3), the patients divided into three groups:

- Euthyroid group (who has average TSH, T3 and T4 levels).

- Hypothyroid group (including clinical and subclinical; who have elevated TSH level).

- Hyperthyroid group (including clinical and subclinical; who have elevated T3 and T4 levels).

\subsection{Inclusion criteria}

- Patients with pathologically proved Hashimoto thyroiditis (cases)

- Healthy control participants.

\subsection{Exclusion criteria}

- Acute thyroid inflammatory conditions.

- Medications that may alter thyroid hormones (Amiodarone, Lithium, Interferons (IFN), Tyrosine Kinase Inhibitors (TKIs), Iodine-containing medications and agents).

- Radioiodine therapy.

- Pregnancy.

\subsection{Methods}

All participants included in the study subjected to the following:

1. Clinical history (age, sex and complain):

Cases revered by their clinician \& clinical history was taken by the radiologist. Most of cases com- plained of neck swelling (40 case), female cases presented with menstrual irregularity (12 case). Other cases presented with dysphagia ( 9 cases), toxic symptoms "palpitation, weight loss, anxiety...." (11 case), easy fatigability (12 cases) \& rest of cases (10 cases) referred due to goitre diagnosed by clinical examination.

\section{Investigations}

\section{A. Radiological:}

Ultrasound examination was performed by radiologist using $10 \mathrm{MHz}$ or higher linear probe (Toshiba, Aplio- Mxssa-780A) and seven sonographic features were analysed for evaluation of Hashimoto thyroiditis, including:

1. Parenchymal echogenicity compared with adjacent strap muscles \& was classified into three grades:

- Grade 0: normal parenchymal echogenicity, which is higher than strap muscles.

- Grade 1: reduced parenchymal echogenicity, which is equal to strap muscles (Fig. 1).

- Grade 2: reduced parenchymal echogenicity, which is less than strap muscles (Fig. 2).

2. Parenchymal micronodularity, which is parenchymal hypoechoic micronodules less than $3 \mathrm{~mm}$ scattered within the thyroid gland with mainly peripheral predominance, cases classified into cases with no parenchymal micronodularity \& cases with parenchymal micronodularity.

3. Parenchymal septations: which are regions of linear echogenic bands (Fig. 2).

4. Thyroid nodules: which measures more than $3 \mathrm{~mm}$. Cases classified into cases with no sizable nodules and cases with nodules (further classified according to Thyroid Imaging Reporting and Data System “TIRADS” classification) (Fig. 3).

5. Undulation of gland margin (which is irregularity of gland surface) Cases classified in to cases with gland undulation and cases with no undulation (Fig. 1).

6. Glandular vascularity: cases classified into cases with normal gland vascularity \& cases with increased gland vascularity (Figs. 2, 4). 


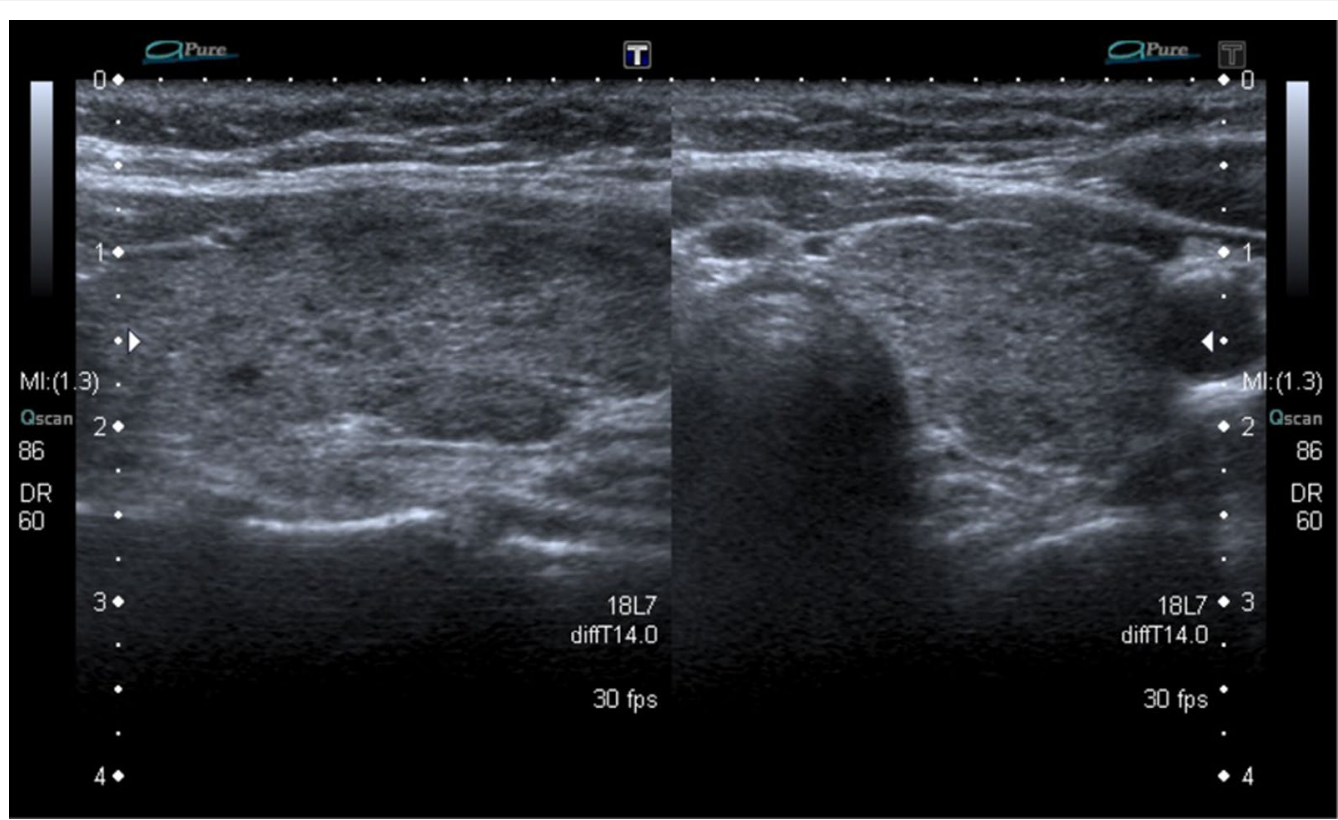

Fig. 1 Female euthyroid patient, 23-years old. Right thyroid lobe longitudinal (right) and transverse (left) views gray-scale U/S shows parenchymal echogenicity grade 1 with undulation of the gland surface

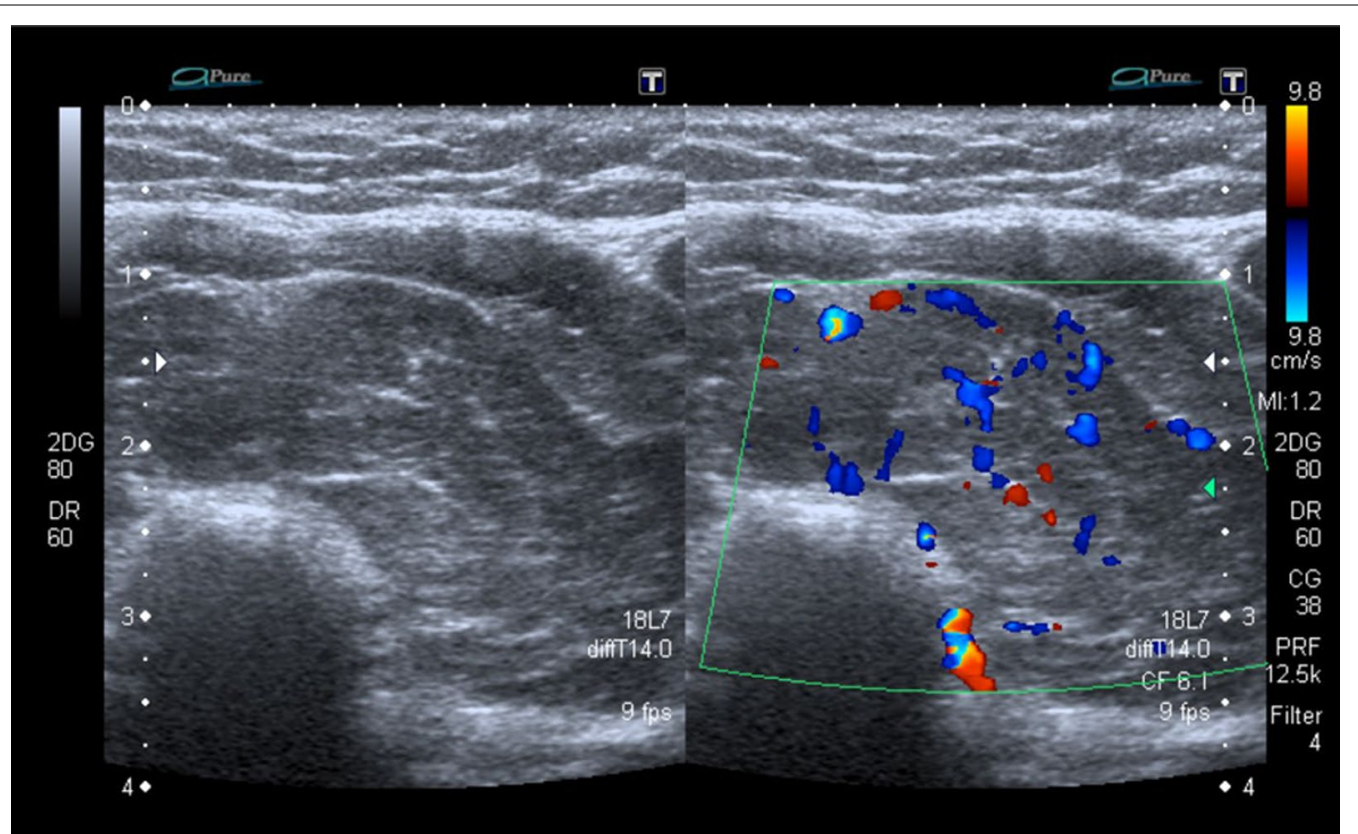

Fig. 2 Male hypothyroid patient, 22-years old. Left thyroid lobe transverse view U/S (gray scale and Doppler) shows parenchymal echogenicity grade 2 , septations with undulation of the gland surface (right), mild increased gland vascularity (left) 


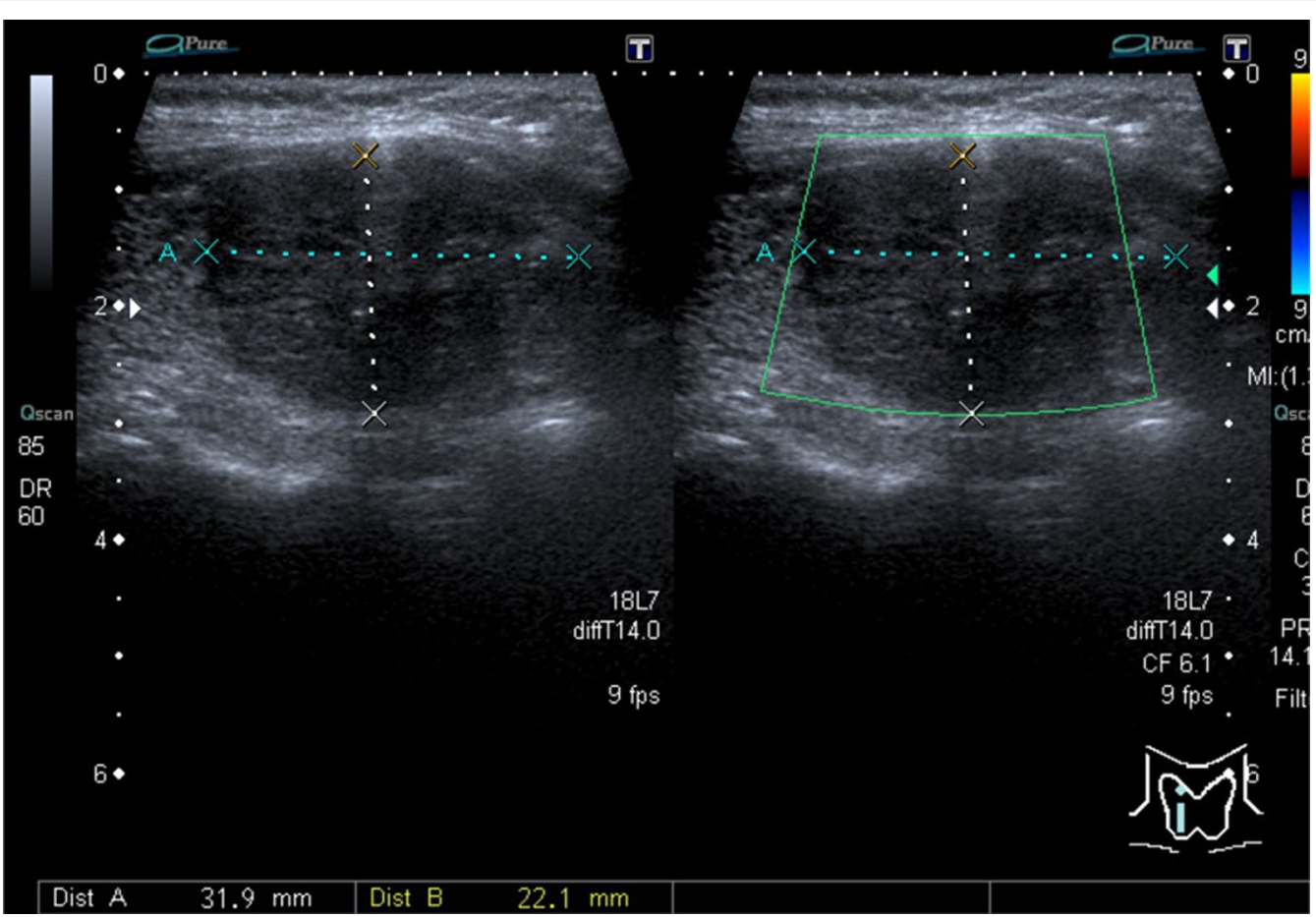

Fig. 3 Male euthyroid patient, 34-years old. Right thyroid lobe longitudinal view; gray-scale (right) and Doppler (left) U/S shows hypoechoic nodules proved to be papillary carcinoma

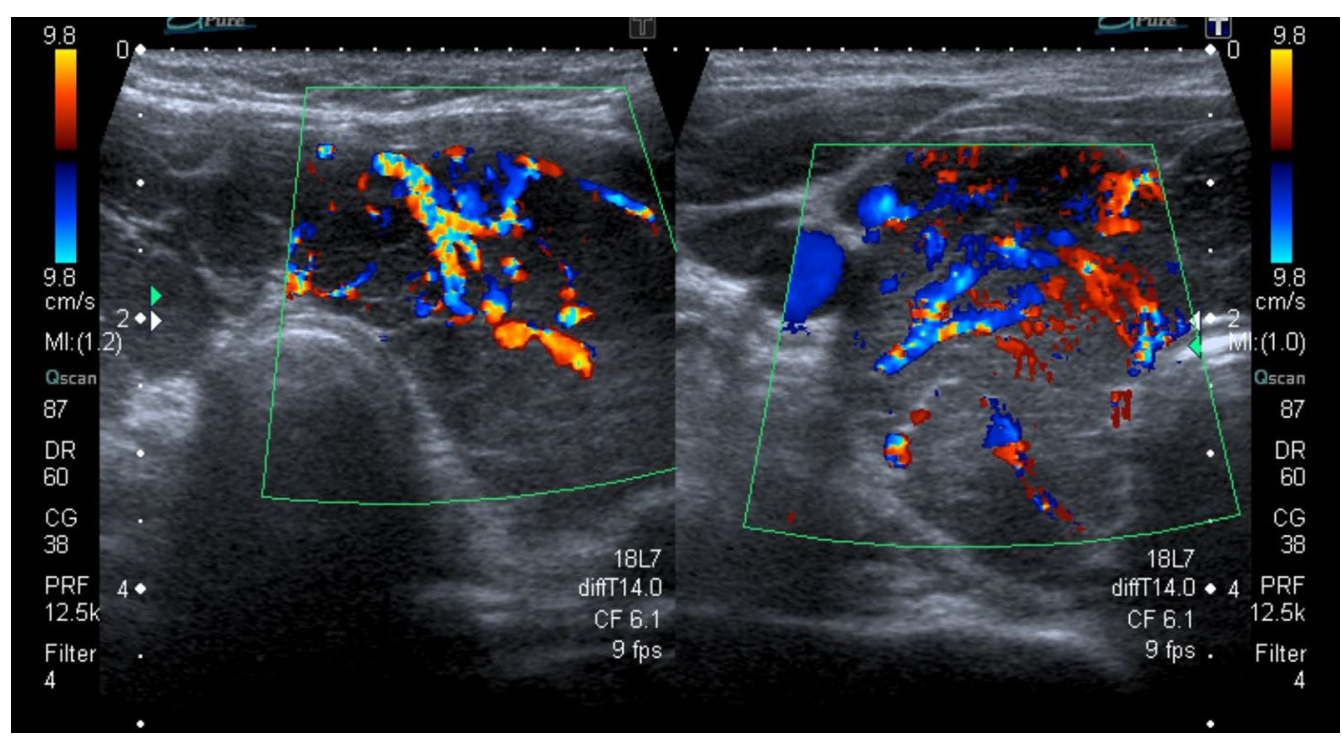

Fig. 4 Female hypothyroid patient, 27-years old. thyroid gland transverse view Doppler U/S shows parenchymal echogenicity grade 2 with evident increased gland vascularity 


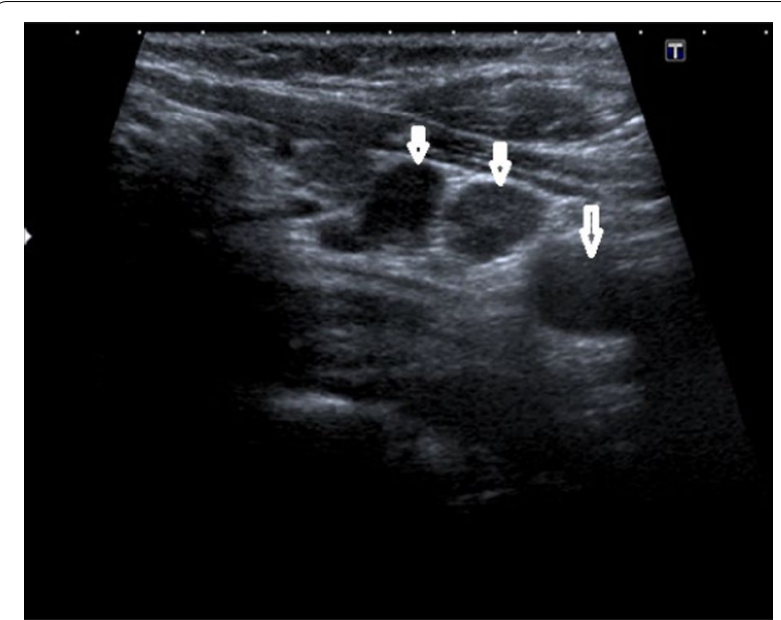

Fig. 5 Female hypothyroid patient, 34-years old. Longitudinal view gray-scale U/S of lower anterior neck region in shows enlarged para-tracheal lymph nodes with globular shape \& lost hilum "arrows"

7. Para-tracheal enlarged lymph nodes: cases classified into cases with enlarged Para-tracheal lymph nodes \& cases with no enlarged Para-tracheal enlarged lymph nodes (Fig. 5).

\section{B. Laboratory:}

Serum levels of TSH, T3 \& T4 were measured using radioimmunoassay methods; Normal T4 level ranged from 4.5 to $12 \mu \mathrm{g} / \mathrm{dL}$ and normal T3 level $0.92-$ $2.79 \mathrm{nmol} / \mathrm{L}$. Serum (TSH) was measured by immuneradiometric assay (normal ranges: 0.3-3.6 mIU/L).

\subsection{Statistical analysis}

Data were collected then entered analysis of data was performed using SPSS v. 25 (Statistical Package for Social science) for Windows (IBM Corp. Released 2017. IBM SPSS Statistics for Windows, Version 25.0. Armonk, NY: IBM Corp.). Description of quantitative variables was presented in the form of mean, standard deviation (SD), median (for nonparametric variables). Description of qualitative variables was presented in the form of numbers (No.) and percent's (\%). Independent T-test was used to detect the difference between cases and controls regarding scale variables. Chi-Squared test was used to detect the difference between both groups regarding the categorical variables. The significance of the results was assessed in the form of $P$-value that was differentiated into; non-significant when $P$ value $>0.05$ and significant when $P$ value $\leq 0.05$.

\section{Results}

Ninety-six pathologically proved cases of Hashimoto thyroiditis ( $8 \%$ males \& 92\% females, main age 36 year) were compared to 100 control cases (32\% males \& $77 \%$ females: main age 39 year). Forty-two percent (42\%) of cases presented with anterior neck swelling, 13\% presented with menstrual irregularity, $12 \%$ with easy fatigability \& $11 \%$ revered by their clinician for goitre noticed in clinical examination.

The thyroid hormonal status of studied cases was fortyfour percent (44\%) of cases had hypothyroidism, (35\%) were euthyroid and (21\%) had hyperthyroidism.

Thyroid gland sonographic parenchymal abnormalities (septations, undulation, micronodularity, gland hyper vascularity, hypoechogenicity, sizable nodules as well as enlarged para-tracheal lymph nodes) were significantly higher in Hashimoto cases compared to control group. Undulation of thyroid gland margin was the most sensitive sonographic sign seen in about $72 \%$ of studied cases while seen only in (4\%) of control group, Parenchymal micronodularity seen an about (71\%) of cases and not seen in control group. Septations noted in (50\%) of cases while seen only in (2\%) of control group. Regarding parenchymal echogenicity about (28\%) of cases showed grade 0 , about (47\%) grade 1 and about (25\%) grade 2 .

Pathologically enlarged para-tracheal lymph nodes found only in about twenty-seven percent $(27 \%)$ of cases $\&$ not seen in control group ( $P$ value is $<0.001)$.

About fifty-eight percent (58\%) of cases showed increased thyroid parenchymal vascularity, while rest of cases showed normal vascularity.

Sizable thyroid nodules found only in twenty-four percent $(24 \%)$ of cases, which significantly higher in cases compared to control group. One nodule (4\% of cases) pathologically proved to be papillary carcinoma, while rest of nodules $(22 / 23)$ classified according to TIRADS classification ( 6 cases TIRADS 2, 15 cases TIRADS $3 \& 1$ case TIRADS 4) \& follow-up recommended. On followup all showed stationary course.

We correlated thyroid hormonal levels with sonographic signs;

- TSH level was significantly high in cases with grade 2 echogenicity compared to cases with grade 0 \& grade 1 echogenicity (Table 1 ).

- TSH \& T4 levels were significantly high in cases with high gland vascularity in Doppler study (Table 2).

- TSH level was significantly high in cases with para-tracheal abnormal enlarged lymph nodes. (Table 3). 
Table 1 Relation between thyroid gland echogenicity and thyroid hormonal level in studied cases

\begin{tabular}{|c|c|c|c|c|c|}
\hline $\mathrm{Hr}$ & Echogenicity* & Number of cases & Mean \pm SD & Median (IQR) & $P$-value\# \\
\hline \multirow[t]{3}{*}{ T3 } & 0 & 4 & $4.4 \pm 3.7$ & $3.5(3-4.3)$ & 0.485 \\
\hline & 1 & 5 & $3.8 \pm 2.1$ & $3.2(3-4.2)$ & \\
\hline & 2 & 1 & $3.5 \pm 1.2$ & $3.5(3-3.85)$ & \\
\hline \multirow[t]{3}{*}{ T4 } & 0 & 1 & $1.5 \pm 1.4$ & $1(0.9-1.5)$ & 0.912 \\
\hline & 1 & 3 & $1.5 \pm 1$ & $1(0.9-1.6)$ & \\
\hline & 2 & 2 & $1.4 \pm 1.2$ & $1.1(0.63-1.5)$ & \\
\hline \multirow[t]{3}{*}{ TSH } & 0 & 17 & $5.7 \pm 6.2$ & $4(1.1-6.9)$ & $\begin{array}{l}P 0 \text { versus } 1=0.125 \\
P 0 \text { versus } 2=0.999 \\
* P 1 \text { versus } 2=0.018\end{array}$ \\
\hline & 1 & 21 & $3 \pm 3.7$ & $2.15(0.02-3.8)$ & \\
\hline & 2 & 11 & $14.5 \pm 26.4$ & $4.6(2.4-7.6)$ & \\
\hline \multirow[t]{3}{*}{ Euthyroids cases } & 0 & 6 & & & \\
\hline & 1 & 15 & & & \\
\hline & 2 & 10 & & & \\
\hline total & & 96 case & & & \\
\hline
\end{tabular}

\# Kruskal-Wallis test was used as the variables were not normally distributed (Tukey post hoc was used to compare each 2 categories)

*P-value is significant

Table 2 Relation between high thyroid gland vascularity and thyroid hormonal level in studied cases:

\begin{tabular}{|c|c|c|c|c|c|}
\hline hormones & High vascularity & Number of cases & Mean \pm SD & Median (IQR) & $P$-value\# \\
\hline \multirow[t]{2}{*}{ T3 } & No & 4 & $3.6 \pm 1.1$ & $3.2(3-3.9)$ & 0.225 \\
\hline & Yes & 9 & $4.2 \pm 3.2$ & $3.5(3-4.1)$ & \\
\hline \multirow[t]{2}{*}{ T4 } & No & 2 & $1.2 \pm 0.8$ & $1(0.7-1.2)$ & $0.047^{*}$ \\
\hline & Yes & 3 & $1.6 \pm 1.4$ & $1.1(0.9-1.6)$ & \\
\hline \multirow[t]{2}{*}{$\mathrm{TSH}$} & No & 18 & $3.5 \pm 5.3$ & $1.7(0.4-3.9)$ & $0.030^{*}$ \\
\hline & Yes & 25 & $8.8 \pm 18.1$ & $4.2(0.7-6.8)$ & \\
\hline \multicolumn{6}{|c|}{ Euthyroids cases } \\
\hline & No & 16 & & & \\
\hline & Yes & 19 & & & \\
\hline Total & & 96 case & & & \\
\hline
\end{tabular}

\# Mann-Whitney $\mathrm{U}$ test was used as the variables were not normally distributed (Tukey post hoc was used to compare each 2 categories)

*P-value is significant

Table 3 Relation between presence of para-tracheal abnormal enlarged lymph nodes and thyroid hormonal level in studied cases:

\begin{tabular}{|c|c|c|c|c|c|}
\hline & Enlarged lymph nodes & Number of cases & Mean \pm SD & Median (IQR) & $P$-value\# \\
\hline \multirow[t]{2}{*}{ T3 } & No & 9 & $4.1 \pm 2.8$ & $3.5(3-4.2)$ & 0.408 \\
\hline & Yes & 3 & $3.4 \pm 1$ & $3.3(3-4)$ & \\
\hline \multirow[t]{2}{*}{ T4 } & No & 8 & $1.6 \pm 1.4$ & $1(0.9-1.5)$ & 0.397 \\
\hline & Yes & 2 & $1.1 \pm 0.5$ & $1(0.7-1.4)$ & \\
\hline \multirow[t]{2}{*}{ TSH } & No & 27 & $4.8 \pm 9.9$ & $2.3(0.2-5.5)$ & $0.017^{*}$ \\
\hline & Yes & 13 & $11.5 \pm 21.9$ & $4.5(2.6-6.9)$ & \\
\hline \multirow[t]{2}{*}{ Euothyroid } & No & 25 & & & \\
\hline & Yes & 9 & & & \\
\hline Total & & 96 case & & & \\
\hline
\end{tabular}

\# Mann-Whitney $U$ test was used as the variables were not normally distributed. (Tukey post hoc was used to compare each 2 categories)

${ }^{*} P$-value is significant 
Hormonal levels not significantly correlated with other sonographic signs.

\section{Discussion}

Hashimoto thyroiditis (HT) is a chronic autoimmune thyroid gland inflammation [5] it considered the most common endocrine disorder [6] and the most common cause of hypothyroidism [7].

In this study, we retrospectively studied 96 pathologically proved cases of Hashimoto thyroiditis (8\% males \& $92 \%$ females, main age 36 year) \& compared them with 100 -control case $(32 \%$ males \& $77 \%$ females: main age 39 year). We reported that HT is significantly more common in females. This was similar to many previous studies $[8,15,16]$.

About $8 \%$ of cases ( 8 of 96 ) are below 20 years, four of them below 15 years. This matches with studies done by Januś et al. [17], Zdraveska et al. [18], Zois et al. [19], all reported that prevalence of chronic type of autoimmune thyroiditis has been assessed as $4-9.6 \%$ in adolescents.

Regarding laboratory assessment of study cases, we reported that most of cases (44\%) were hypothyroid, (35\%) euthyroid \& $21 \%$ hyperthyroid. The majority of cases being in hypothyroidism state matches what stablished about HT to be the most common cause of hypothyroidism [2, 16, 20]. In study done by Staii et al. [9] they reported that only $6 \%$ of HT cases showed clinical hypothyroidism and $46 \%$ of HT cases show euthyroid, compared to only (36\%) of our study cases, this could be explained by in cases showing euthyroidism with extrathyroid manifestations, clinicians not frequently refer them for thyroid ultrasound.

Sonographic features of Hashimoto thyroiditis are established \& discussed in many studies $[8,14,15,21]$. These features include enlarged thyroid gland, parenchymal micronodularity, parenchymal septations, parenchymal hypo-echogenicity, surface Undulation as well as altered vascularity.

We used these sonographic criteria in our study to compare patients with HT \& control cases. In the present study, Thyroid gland's sonographic parenchymal abnormalities (septations, undulation of gland surface \& micronodularity) were significantly higher in Hashimoto cases compared to control group. Parenchymal hypo echogenicity \& undulation of thyroid gland margin were the most sensitive sonographic signs seen in about $72 \%$ of studied cases (69/96) followed by Parenchymal micronodularity seen in about 71\% (68/96). Parenchymal septations seen only in $50 \%$ of cases $(48 / 96)$. The least sensitive sign is the presence of sizable nodule seen only in $24 \%$ of cases (23/96). This was comparable to study done by Patel et al. [15] who reported that parenchymal heterogeneity and diffuse hypoechogenicity were the most sensitive sonographic features of HT.

In our study, about $73 \%$ of cases show parenchymal hypo echogenicity " $47 \%$ grade $1 \& 25 \%$ grade 2. " This matches with Pedersen et al. [10], Schiemann et al. [11] and Loy et al. [12], Who reported a strong relationship between gland hypoechogenicity and HT. According to their studies, parenchymal hypoechogenicity is an index reflecting the degree of gland autoimmune involvement. We support this theory as well as it may be due to element of gland fibrosis secondary to chronic inflammatory process.

In current study TSH level was significantly higher in cases with grade 2 parenchymal echogenicity compared to cases with grad 0 (normal echogenicity) as well as control group. This matches with what reported by Loy et al. [12], who reported that hypothyroid patients have significantly lower parenchymal echogenicity compared with euthyroid healthy group.

Regarding the enlarged para tracheal lymph nodes, it was significantly high in HT group compared to control group, however it found only in 27\% of cases of HT. this mismatches with what reported by SERRES-CRE 'IXAMS, et al. [13] who found that PLNs were seen in 184 of 199 patients in cases with auto-immune thyroiditis group (about 95\%). We found that presence of PLNs is strongly suggestive of HT but not frequently seen, this may be due to increased incidence of PLNs in other autoimmune thyroiditis other than HT, which not included in our study. In our reported cases with PLNs, TSH level was significantly higher compared to cases with normal PLNs \& control group.

The thyroid gland vascularity was significantly higher in cases of HT (about 58\% of cases have increased vascularity) on Doppler study. In such cases, TSH \& T4 levels were significantly higher compared to cases with average vascularity. This means that vascularity is increased in cases with hyper \& hypothyroidism compared to euthyroid cases but not specific to one of them, this was similar to study done by Acar et al. [8] who found that the thyroid gland vascularity was higher in patients with hyperthyroidism and in the patients in the hypothyroid state.

Regarding sizable thyroid gland nodules, we reported only 23 cases ( $24 \%$ of cases) having sizable nodules. Four per cent of these nodules $(1$ cases/23) pathologically proved to be malignant (papillary carcinoma) and remaining 22 cases classified according to TIRADS classification ( 6 cases TIRADS 2, 15 cases TIRADS 3 and 1 case TIRADS 4) this may be partially matches with study done by Anderson et al. [14] who reported that $16 \%$ only of thyroid nodules in HT were malignant (papillary carcinoma \& lymphoma). It also partially similar to study done by Patel et al. [15] who reported that most malignant nodules were papillary carcinoma ( $89 \%$ of all malignancies), yet no matches with them in reporting that 
(44\% of cases with nodules) were malignant, but this may be caused by their study group were suspected to have malignancy from the start. Additionally, our study lake of histopathological examination for nodules subjected by TIRADS classification to follow-up not biopsy. On follow-up (22 from 22 cases) show stationary course.

Limitation of our study was the small sample size \& being single centre study.

\section{Conclusions}

Ultrasound is a very good diagnostic tool in HT with established signs. It can suggest hypothyroidism (high TSH) in cases of grade 2 parenchymal echogenicity, high vascularity \& para-tracheal lymph nodes enlargement. These finding could be helpful in patient management $\&$ can contribute in better understanding the disease progression.

\section{Abbreviations} \\ HT: Hashimoto thyroiditis.; US: Ultrasound.; TSH: Thyroid stimulating hormone.; \\ T4: Thyroxine; T3: Triiodothyronine; PLNs: Para-tracheal lymph nodes; TIRADS: \\ Thyroid imaging reporting and data system; PACS: Picture archiving and com- \\ munication system.
}

\section{Acknowledgments}

Not applicable.

\section{Authors' contributions}

RM collected data and wrote the manuscript. KA, FM, and AS revised collected data, did statistical analysis and revised the manuscript. All authors have read and approved the final manuscript.

\section{Funding}

None.

\section{Availability of data and materials}

All data and materials are available.

\section{Declarations}

\section{Ethics approval and consent to participate}

The study protocol subjected to approval by the ethical committee of the Faculty of Medicine in Beni-Suef University (approval No: FMBSURE/06122020/ Mohammed). Consent to participate is not applicable since it is a retrospective study that was done using saved data on (PACS) picture archiving \& communication system.

\section{Consent for publication}

Written informed consent for publication was obtained from all patients.

\section{Competing interests}

The authors declare that they have no competing interests.

\section{Author details}

${ }^{1}$ Radiology Department, Bani-Seuf University, Bani Seuf, Egypt. ${ }^{2}$ Plastic Surgery Department, Bani-Seuf University, Bani Seuf, Egypt. ${ }^{3}$ General Surgery Department, Bani-Seuf University, Bani Seuf, Egypt.

Received: 25 November 2021 Accepted: 11 January 2022

Published online: 23 February 2022

\section{References}

1. Yildirim D, Gurses B, Gurpinar B, Ekci B, Colakoglu B, Kaur A (2011) Nodule or pseudonodule? Differentiation in Hashimoto's thyroiditis with sonoelastography. J Int Med Res 39:2360-2369

2. Dighe M, Barr R, Bojunga J, Cantisani V, Chammas MC, Cosgrove D (2017) Thyroid ultrasound: state of art part 1 -thyroid ultrasound reporting and diffuse thyroid disease. Med Ultrason 19(1):79-93. https://doi.org/10. 11152/mu-980

3. Ceylan I, Yener S, Bayraktar F, Mustafa S (2014) Roles of ultrasound and power Doppler ultrasound for diagnosis of Hashimoto thyroiditis in anti-thyroid marker-positive euthyroid subjects. Quant Imaging Med Surg 4(4):232-238

4. Kim DW, Eun CK, In HS, Kim MH, Jung SJ, Bae SK (2010) Sonographic differentiation of asymptomatic diffuse thyroid disease from normal thyroid: a prospective study. AJNR Am J Neuroradiol 31:1956-1960

5. McLeod DS, Cooper DS (2012) The incidence and prevalence of thyroid autoimmunity. Endocrine 42:252-265

6. Golden SH, Robinson KA, Saldanha I, Anton B, Ladenson PW (2009) Clinical review: prevalence and incidence of endocrine and metabolic disorders in the United States: a comprehensive review. J Clin Endocrinol Metab 94:1853-1878

7. Vanderpump MP (2011) The epidemiology of thyroid disease. Br Med Bull 99:39-51

8. Acar T, Özbek SS, Erdoğan M, Özgen AG, Demirel SO (2013) US findings in euthyroid patients with positive antithyroid autoantibody tests compared to normal and hypothyroid cases. Diagn Interv Radiol 19:265-270

9. Staii A, Mirocha S, Todorova-Koteva K, Glinberg S, Jaume JC (2010) Hashimoto thyroiditis is more frequent than expected when diagnosed by cytology which uncovers a pre-clinical state. Thyroid Res 3:11

10. Pedersen OM, Aardal NP, Larssen TB, Varhang JE, Myking O, Vik-Mo H (2000) The value of ultrasonography in predicting autoimmune thyroid disease. Eur Thyroid J 10:251-259

11. Loy M, Cianchetti ME, Cardia F, Melis A, Boi F, Mariotti S (2004) Correlation of computerized gray-scale sonographic findings with thyroid function and thyroid autoimmune activity in patients. J Clin Ultrasound 32:136-140

12. Schiemann U, Avenhaus W, Konturek JW, Gellner R, Hengst K, Gross M (2003) Relationship of clinical features and laboratory parameters to thyroid echogenicity measured by standardized grey scale ultrasonography in patients with Hashimoto's hyroiditis. Med Sci Monit 9:13-17

13. Serres-Cre'ixams X, Castells-Fuste I, Pruna-Comella X et al (2008) Paratracheal lymph nodes: a new sonographic finding in autoimmune thyroiditis. J Clin Ultrasound 36(7)

14. Anderson L, Middleton WD, Teefey SA, Reading CC, Langer JE et al (2010) Hashimoto thyroiditis: part 2, sonographic analysis of benign and malignant nodules in patients with diffuse hashimoto thyroiditis. AJR 195(216):222

15. Patel S, Giampoli E, Oppenheimer D, Montoya S, Rupasov A, Dogra V (2018) Sonographic features of diffuse hashimoto thyroiditis: determining sensitivity of features and predictors of malignancy. Am J Sonogr 1(1)

16. Takahashi MS, Moraes PHM, Chammas MC (2019) Ultrasound evaluation of thyroiditis: a review. J Otolaryngol Res 2(1):127

17. Januś D, Wójcik M, Drabik G, Wyrobek Ł,Starzyk JB (2018) Ultrasound variants of autoimmune thyroiditis in children and adolescents and their clinical implication in relation to papillary thyroid carcinoma development. J Endocrinol Invest 41:371-380

18. Zdraveska N, Kocova M (2012) Hashimoto thyroiditis in childhood-review of the epidemiology, genetic susceptibility and clinicalaspects of the disease. Maced J Med Sci 5(3):336-345

19. Zois C, Stavrou I, Kalogera C, Svarna E (2003) High prevalence of autoimmune thyroiditis in school children after elimination of iodine deficiency in northwestern Greece. Thyroid 13:485-489

20. Pearce EN, Farwell AP, Braverman LE (2003) Thyroiditis. N Engl J Med 348:2646-2655

21. Hayashi N, Tamaki N, Konishi J, Yonekura Y, Senda M, Kasagi K (1986) Sonography of Hashimoto's thyroiditis. J Clin Ultrasound 14:123-126

\section{Publisher's Note}

Springer Nature remains neutral with regard to jurisdictional claims in published maps and institutional affiliations. 